\title{
Supplementary
}

\section{Three-Dimensional Resonant Exciton in Monolayer Tungsten Diselenide Actuated by Spin-Orbit Coupling}

Chi Sin Tang ${ }^{1,5, \dagger}$, Xinmao Yin ${ }^{1,3, \dagger, *}$, Ming Yang ${ }^{4, \dagger}, \mathrm{Di}_{\mathrm{Wu}}^{2,6}$, Muhammad Danang Birowosuto ${ }^{9,10}$, Jing $\mathrm{Wu}^{4}$, Changjian $\mathrm{Li}^{8}$, Chathuranga Hettiarachchi ${ }^{10}$, Xin Yu Chin ${ }^{9}$, Yung-Huang Chang7, Fangping Ouyang ${ }^{6}$, Cuong Dang ${ }^{9,10}$, Stephen J. Pennycook ${ }^{8}$, Yuanping Feng ${ }^{1}$, Shijie Wang ${ }^{4}$, Dongzhi Chi ${ }^{4}$, Mark B. H. Breese ${ }^{1,3}$, Wenjing Zhang', Andrivo Rusydi ${ }^{1,3,5}$, Andrew T. S. $\mathrm{Wee}^{1,3,5,{ }^{*}}$.

1. Department of Physics, Faculty of Science, National University of Singapore, 117542, Singapore

2. International Collaborative Laboratory of 2D Materials for Optoelectronics Science and Technology, Shenzhen University, Shenzhen 518060, China

3. Singapore Synchrotron Light Source (SSLS), National University of Singapore, 117603, Singapore

4. Institute of Materials Research and Engineering, A*STAR (Agency for Science, Technology and Research), 2 Fusionopolis Way, 138634, Singapore

5. NUS Graduate School for Integrative Sciences and Engineering, National University of Singapore, 117456, Singapore

6. School of Physics and Electronics, Central South University, No. 932, South Lushan Road, Changsha, Hunan Province 410083, China

7. Bachelor Program in Interdisciplinary Studies, National Yunlin University of Science and Technology, 123 University Road, Section 3, Douliou, Yunlin 64002, Taiwan

8. Department of Materials Science \& Engineering, National University of Singapore, 9 Engineering Drive 1, Singapore 117575, Singapore

9. CINTRA UMI CNRS/NTU/THALES 3288, Research Techno Plaza, 50 Nanyang Drive, Border X Block, Level 6, Singapore 637553, Singapore

10. School of Electrical and Electronic Engineering, Nanyang Technological University, 50 Nanyang Avenue, Singapore 639798, Singapore 


\section{Supplementary Figures}

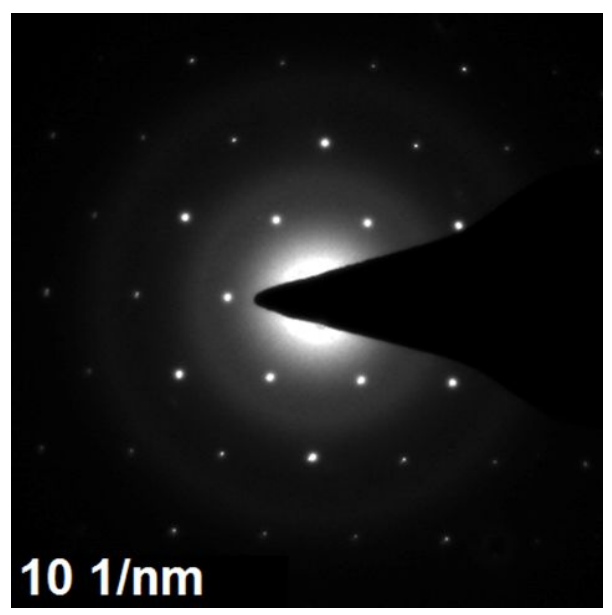

Figure S1. Selected area electron diffraction (SAED) image of the 1H-phase monolayer$\mathrm{WSe}_{2}$ sample.
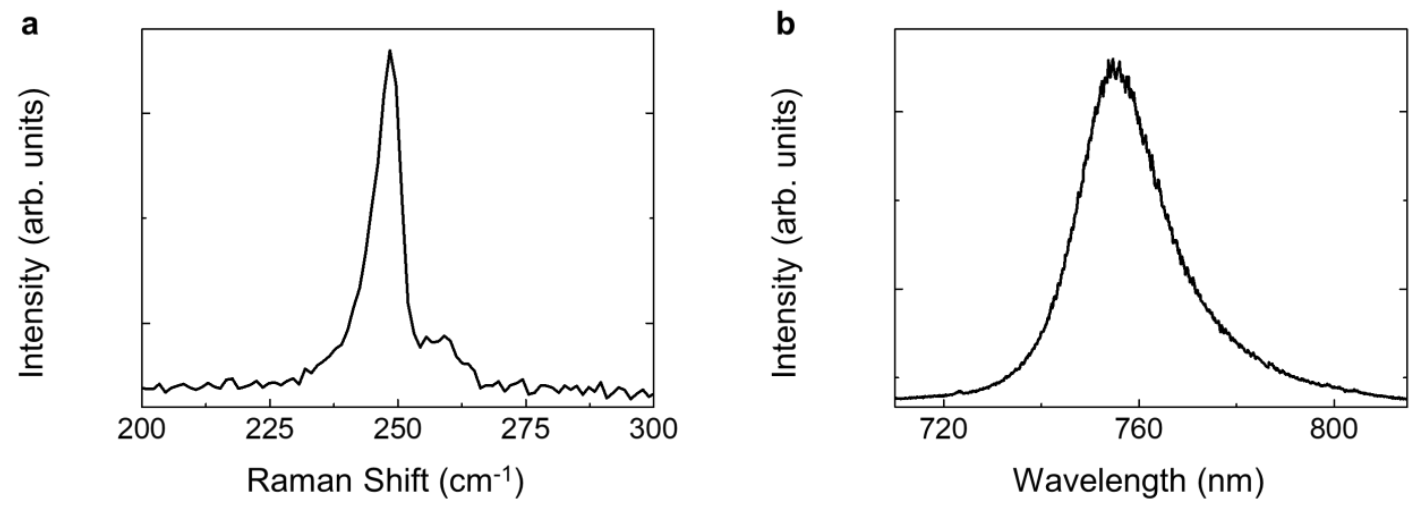

Figure S2. Raman and Photoluminescence spectra for the CVD monolayer-WSe $\mathrm{W}_{2}$ on sapphire. a Raman spectra, where the two characteristic peaks for monolayer-WSe $\mathrm{S}_{2}$ at $\sim 250 \mathrm{~cm}^{-1}\left(E^{1}{ }_{2 \mathrm{~g}}\right.$-mode), and $\sim 261 \mathrm{~cm}^{-1}\left(A_{1 \mathrm{~g}}\right.$-mode). b Photoluminescence spectra, a strong emission at $\sim 755 \mathrm{~nm}$ which corresponds to the A excitonic absorption of monolayer$\mathrm{WSe}_{2}$. 

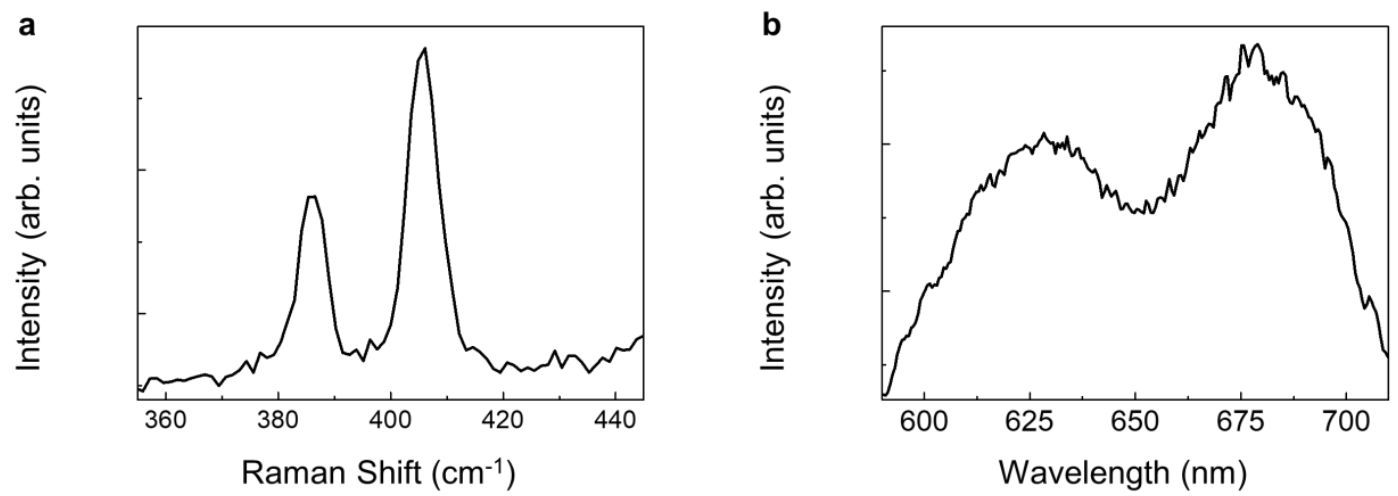

Figure S3. Raman and Photoluminescence spectra for the CVD monolayer- $\mathrm{MoS}_{2}$ on sapphire. a Raman spectra, where the two characteristic peaks for monolayer-MoS $\mathrm{S}_{2}$ at $\sim 395 \mathrm{~cm}^{-1}\left(E^{1}{ }_{2 \mathrm{~g}}\right.$-mode), and $\sim 415 \mathrm{~cm}^{-1}\left(A_{1 \mathrm{~g}}\right.$-mode). b Photoluminescence spectra, strong emissions at $\sim 673 \mathrm{~nm}$ and $\sim 620 \mathrm{~nm}$ corresponding to the excitonic absorptions $\mathrm{A}$ and $\mathrm{B}$, respectively for monolayer- $\mathrm{MoS}_{2}$.

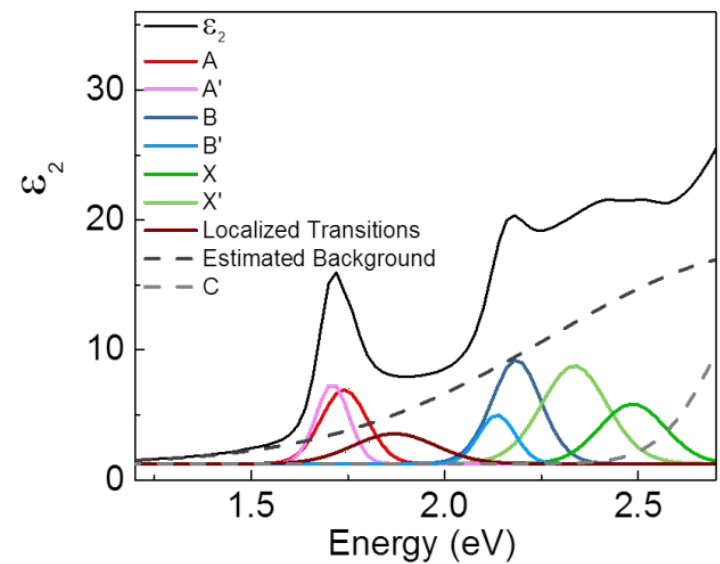

Figure S4. $\varepsilon_{2}$ spectrum from spectroscopic ellipsometry at $4 \mathrm{~K}$ with contributions from the respective excitons and the estimated defect and background signals. Note the peak feature at the estimated position of $\sim 1.87 \mathrm{eV}$ (labelled 'Localized Transitions') is added to account for the combination of effects due to excited excitons and localized transitions which have previously been observed in monolayer- $\mathrm{WSe}_{2}{ }^{1-2}$. 


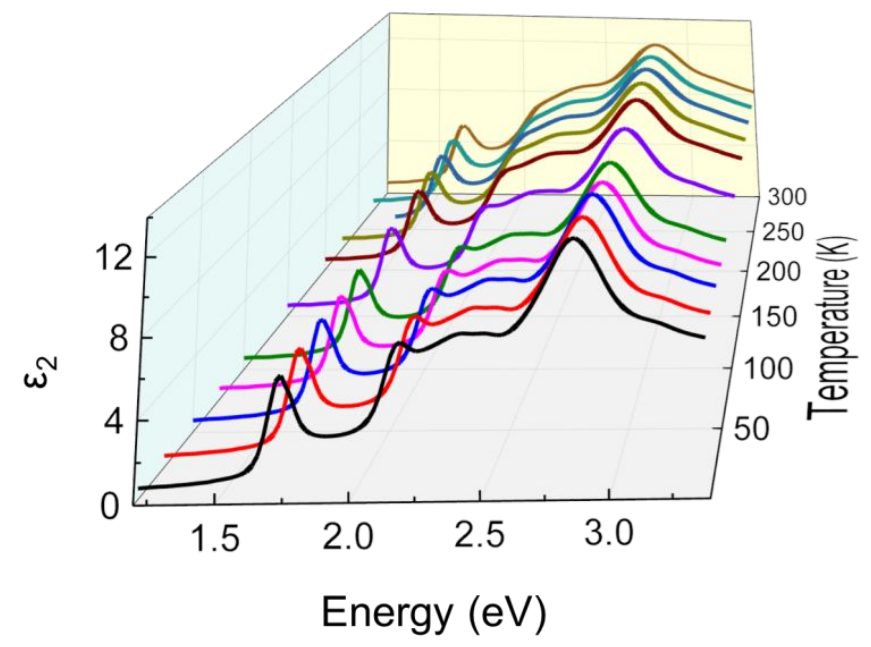

Figure S5. $\varepsilon_{2}$ spectra of $1 \mathrm{H}$-phase monolayer-WSe $\mathrm{W}_{2}$ throughout the temperature range $4-$ $300 \mathrm{~K}$.

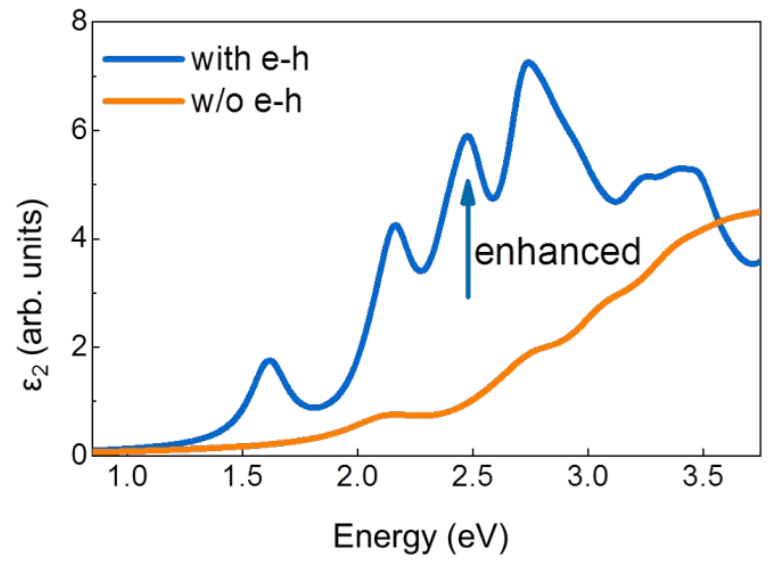

Fig. S6. First-principles calculation comparing $\varepsilon_{2}$ spectra of monolayer-WSe $\mathrm{Se}_{2}$ with and without the electron-hole interaction effects. The RPA (w/o e-h) spectrum that has been redshifted by $0.5 \mathrm{eV}$ has been included as main text Fig. $2 \mathrm{f}$. 


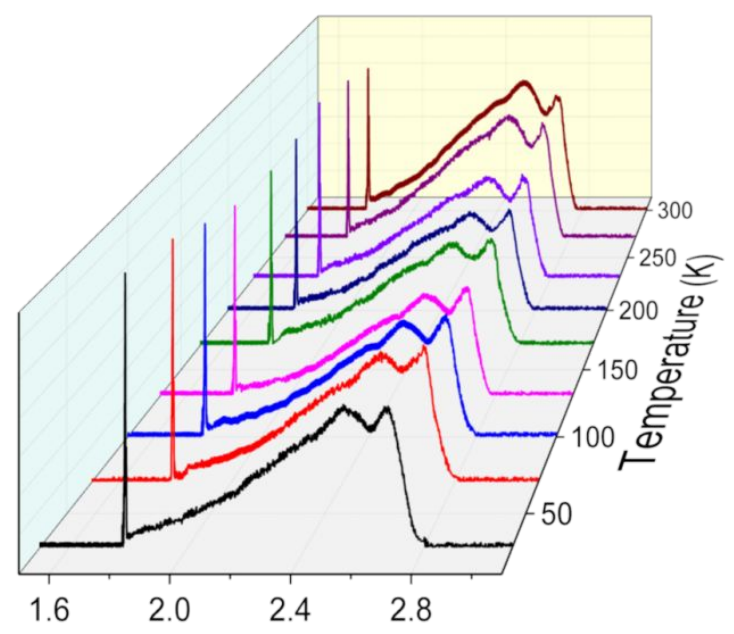

Fig. S7. Waterfall image of the temperature-dependent photoluminescence spectra for monolayer-WSe . $^{2}$
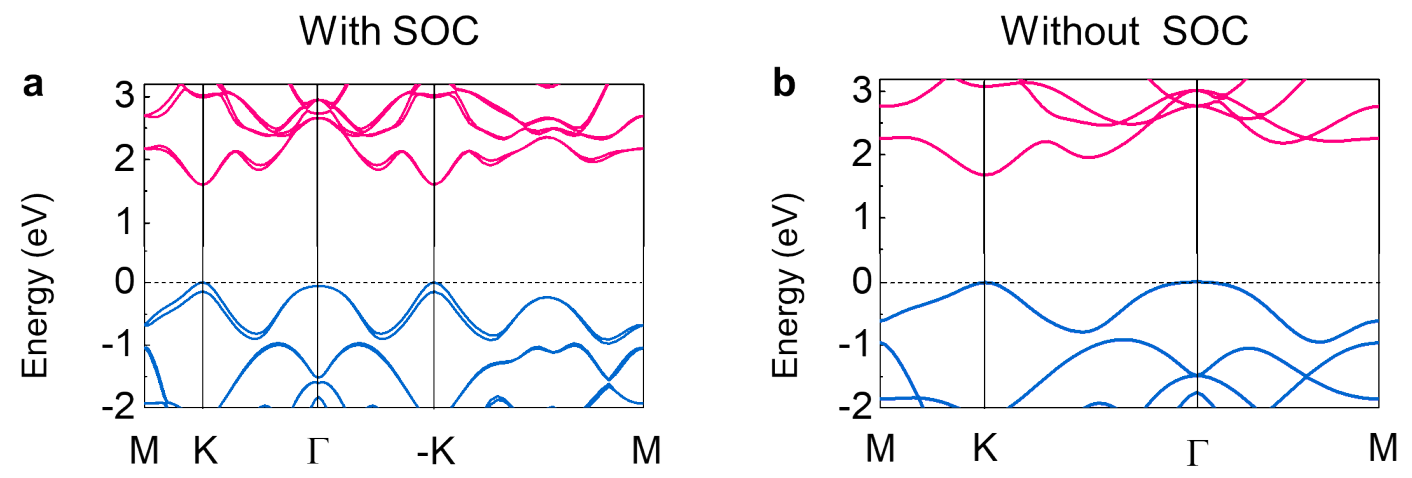

Fig. S8. a Electronic band structure of monolayer-MoS $\mathrm{S}_{2}$ with and, b without the effects of spin-orbit coupling. The dashed black lines in the diagrams serve as visual guides indicating the position of the Fermi level. 

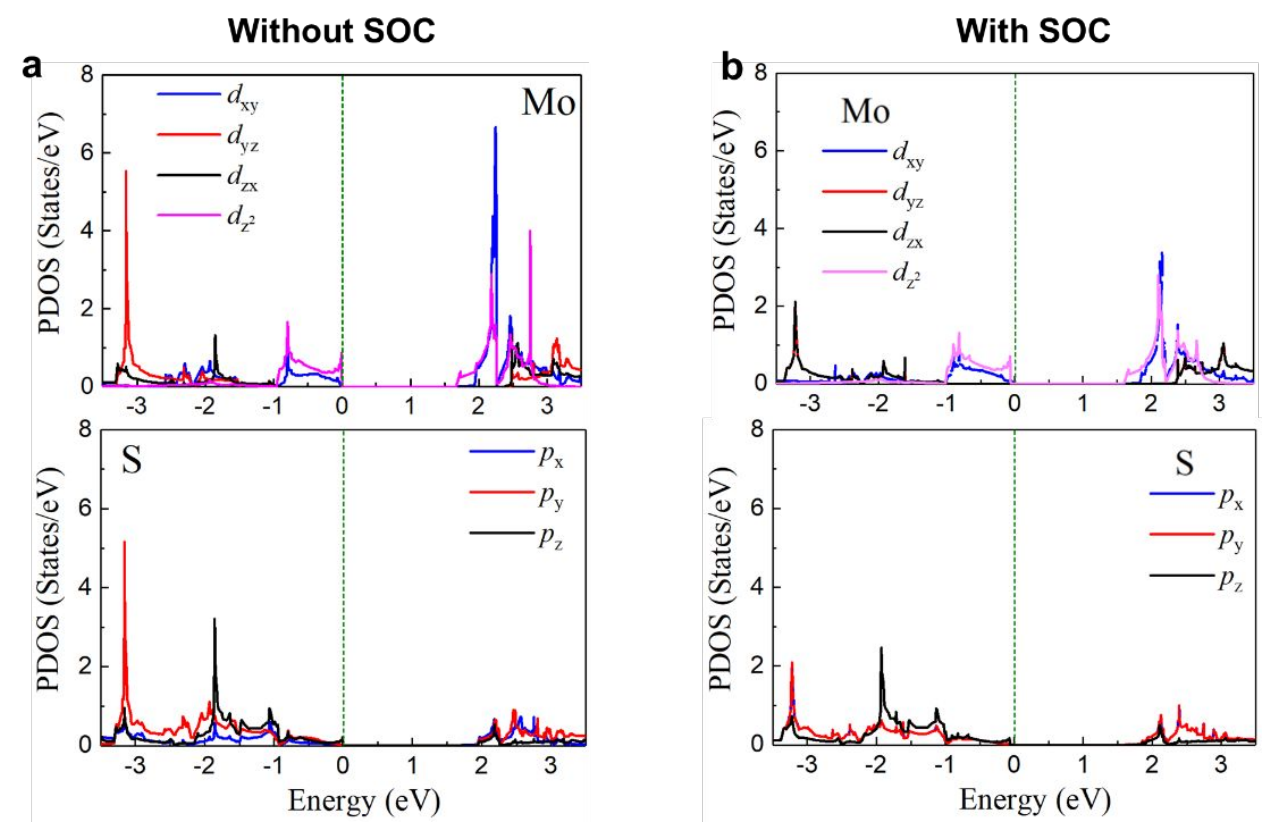

Figure S9. Partial Density of States (PDOS). a Upper panel: PDOS of Mo-atom and, Lower Panel: S-atom without the effects of spin-orbit coupling. b Upper panel: PDOS of Mo-atom and, Lower Panel: S-atom which include the effects of spin-orbit coupling.

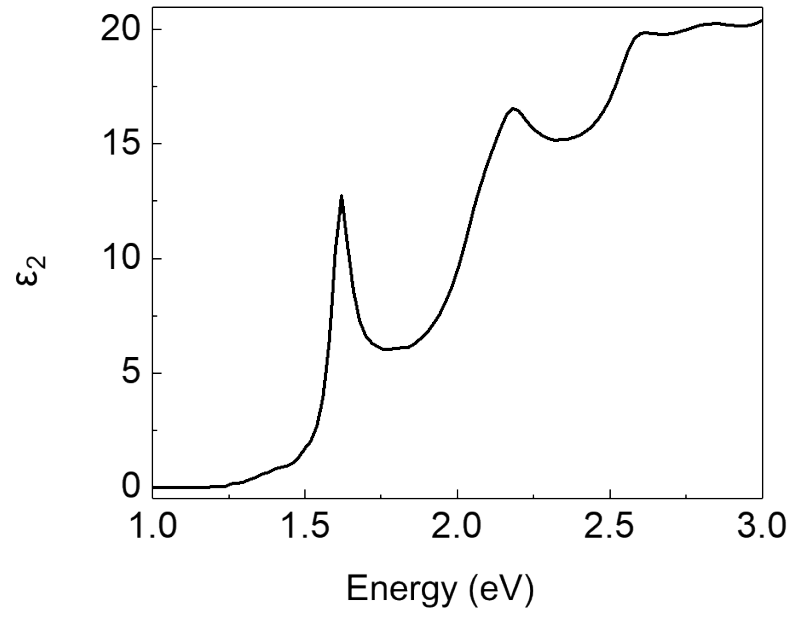

Figure S10. $\varepsilon_{2}$ spectra of $1 \mathrm{H}$-phase bulk $\mathrm{WSe}_{2}$ at $300 \mathrm{~K}$. 


\section{Supplementary Tables}

\begin{tabular}{|c|c|c|c|c|}
\hline Temp (K) & $\begin{array}{c}\text { A Position } \\
\mathbf{e V}\end{array}$ & $\begin{array}{c}\text { A Width } \\
\mathbf{e V}\end{array}$ & $\begin{array}{c}\mathbf{A}^{\prime} \text { Position } \\
\mathbf{e V}\end{array}$ & $\begin{array}{c}\mathbf{A}^{\prime} \text { Width } \\
\mathbf{e V}\end{array}$ \\
\hline 4 & $1.735(7)$ & $0.134(5)$ & $1.690(7)$ & $0.090(2)$ \\
\hline 25 & $1.728(6)$ & $0.135(5)$ & $1.671(5)$ & $0.090(2)$ \\
\hline 50 & $1.727(5)$ & $0.137(5)$ & $1.670(8)$ & $0.091(2)$ \\
\hline 77 & $1.724(7)$ & $0.135(3)$ & $1.669(9)$ & $0.092(2)$ \\
\hline 100 & $1.717(7)$ & $0.138(4)$ & $1.669(9)$ & $0.092(1)$ \\
\hline 125 & $1.710(8)$ & $0.140(6)$ & $1.669(7)$ & $0.093(2)$ \\
\hline 150 & $1.705(5)$ & $0.141(3)$ & $1.665(7)$ & $0.095(3)$ \\
\hline 175 & $1.695(7)$ & $0.142(6)$ & $1.650(7)$ & $0.096(2)$ \\
\hline 200 & $1.689(9)$ & $0.149(4)$ & $1.645(7)$ & $0.097(3)$ \\
\hline 225 & $1.682(6)$ & $0.157(4)$ & $1.645(6)$ & $0.097(2)$ \\
\hline 250 & $1.670(6)$ & $0.158(4)$ & $1.620(8)$ & $0.097(1)$ \\
\hline 275 & $1.659(9)$ & $0.161(5)$ & $1.605(8)$ & $0.098(2)$ \\
\hline 300 & $1.653(5)$ & $0.164(4)$ & $1.590(6)$ & $0.098(2)$ \\
\hline
\end{tabular}

Table S1. Profiles of exciton A and associated trion $A^{\prime}$ elucidated from spectroscopic ellipsometry.

\begin{tabular}{|c|c|c|c|c|}
\hline Temp (K) & $\begin{array}{c}\text { B Position } \\
\mathbf{e V}\end{array}$ & $\begin{array}{c}\text { B width } \\
\mathbf{e V}\end{array}$ & $\begin{array}{c}\text { B' Position } \\
\mathbf{e V}\end{array}$ & $\begin{array}{c}\text { B' width } \\
\mathbf{e V}\end{array}$ \\
\hline 4 & $2.185(9)$ & $0.130(6)$ & $2.145(7)$ & $0.090(2)$ \\
\hline 25 & $2.184(8)$ & $0.125(4)$ & $2.140(9)$ & $0.0905(3)$ \\
\hline 50 & $2.184(10)$ & $0.127(6)$ & $2.140(8)$ & $0.0907(2)$ \\
\hline 77 & $2.183(6)$ & $0.129(5)$ & $2.139(8)$ & $0.0913(2)$ \\
\hline 100 & $2.179(8)$ & $0.131(5)$ & $2.120(9)$ & $0.0916(2)$ \\
\hline 125 & $2.175(6)$ & $0.141(6)$ & $2.120(10)$ & $0.0918(2)$ \\
\hline 150 & $2.170(9)$ & $0.141(5)$ & $2.120(7)$ & $0.092(2)$ \\
\hline 175 & $2.159(8)$ & $0.148(4)$ & $2.119(9)$ & $0.092(2)$ \\
\hline 200 & $2.157(7)$ & $0.160(3)$ & $2.105(9)$ & $0.0935(2)$ \\
\hline 225 & $2.155(8)$ & $0.173(9)$ & $2.090(10)$ & $0.0939(2)$ \\
\hline 250 & $2.149(7)$ & $0.177(7)$ & $2.060(8)$ & $0.0944(2)$ \\
\hline 275 & $2.139(9)$ & $0.183(7)$ & $2.055(9)$ & $0.0953(2)$ \\
\hline 300 & $2.137(9)$ & $0.181(8)$ & $2.050(8)$ & $0.0956(2)$ \\
\hline
\end{tabular}

Table S2. Profiles of exciton B and associated trion B' elucidated from spectroscopic ellipsometry. 


\begin{tabular}{|c|c|}
\hline Temp (K) & $\begin{array}{c}\text { A-B Difference } \\
\text { eV }\end{array}$ \\
\hline 4 & $0.449(16)$ \\
\hline 25 & $0.455(14)$ \\
\hline 50 & $0.457(15)$ \\
\hline 77 & $0.458(13)$ \\
\hline 100 & $0.461(15)$ \\
\hline 125 & $0.465(14)$ \\
\hline 150 & $0.464(14)$ \\
\hline 175 & $0.464(15)$ \\
\hline 200 & $0.469(16)$ \\
\hline 225 & $0.473(14)$ \\
\hline 250 & $0.479(13)$ \\
\hline 275 & $0.480(18)$ \\
\hline 300 & $0.484(14)$ \\
\hline
\end{tabular}

Table S3. Energy difference between excitons A and B elucidated from spectroscopic ellipsometry.

\begin{tabular}{|c|c|c|c|c|}
\hline Temp (K) & $\begin{array}{c}\text { X Position } \\
\mathbf{e V}\end{array}$ & $\begin{array}{c}\text { X width } \\
\mathbf{e V}\end{array}$ & $\begin{array}{c}\mathbf{X}^{\prime} \text { Position } \\
\mathbf{e V}\end{array}$ & $\begin{array}{c}\mathbf{X}^{\prime} \text { width } \\
\mathbf{e V}\end{array}$ \\
\hline 4 & $2.495(16)$ & $0.170(6)$ & $2.360(14)$ & $0.170(6)$ \\
\hline 25 & $2.490(16)$ & $0.170(8)$ & $2.355(16)$ & $0.170(7)$ \\
\hline 50 & $2.485(11)$ & $0.171(7)$ & $2.352(19)$ & $0.171(8)$ \\
\hline 77 & $2.481(13)$ & $0.175(6)$ & $2.340(19)$ & $0.175(8)$ \\
\hline 100 & $2.475(15)$ & $0.177(7)$ & $2.341(19)$ & $0.175(8)$ \\
\hline 125 & $2.470(17)$ & $0.179(5)$ & $2.325(18)$ & $0.181(6)$ \\
\hline 150 & $2.465(19)$ & $0.181(9)$ & $2.321(18)$ & $0.181(8)$ \\
\hline 175 & $2.461(21)$ & $0.185(10)$ & $2.318(22)$ & $0.184(9)$ \\
\hline 200 & $2.462(25)$ & $0.189(12)$ & $2.310(27)$ & $0.184(11)$ \\
\hline 225 & $2.455(29)$ & $0.198(13)$ & $2.290(28)$ & $0.187(13)$ \\
\hline 250 & $2.456(32)$ & $0.205(14)$ & $2.285(36)$ & $0.188(15)$ \\
\hline 275 & $2.441(34)$ & $0.213(16)$ & $2.270(39)$ & $0.189(17)$ \\
\hline 300 & $2.401(45)$ & $0.224(18)$ & $2.230(49)$ & $0.190(18)$ \\
\hline
\end{tabular}

Table S4. Profiles of exciton $X$ and associated charged exciton $X$ ' elucidated from spectroscopic ellipsometry.

\begin{tabular}{|c|c|c|c|}
\hline $\begin{array}{c}\text { X Position } \\
\mathbf{e V}\end{array}$ & $\begin{array}{c}\text { X Width } \\
\mathbf{e V}\end{array}$ & $\begin{array}{c}\mathbf{X}^{\prime} \text { Position } \\
\mathbf{e V}\end{array}$ & $\begin{array}{c}\mathbf{X}^{\prime} \text { Width } \\
\mathbf{e V}\end{array}$ \\
\hline $2.541(72)$ & $0.129(7)$ & $2.400(80)$ & $0.133(8)$ \\
\hline
\end{tabular}

Table S5. Profiles of exciton $X$ and associated charged exciton $X$ ' elucidated from PL spectroscopy at $30 \mathrm{~K}$. 


\section{Supplementary Methods}

Sample Preparation. $\mathrm{WSe}_{2}\left(\mathrm{MoS}_{2}\right)$ atomic layers were synthesized on sapphire $\left(\mathrm{Al}_{2} \mathrm{O}_{3}\right)$ surface via Chemical Vapour Deposition (CVD) method using $\mathrm{WO}_{3}\left(\mathrm{MoO}_{3}\right)$ and $\mathrm{Se}(\mathrm{S})$ powders as reactants ${ }^{3-4}$. They are then annealed at $200^{\circ} \mathrm{C}$ to enhance the contact between the film and substrate and to eliminate any existing residues. Raman and Photoluminescence spectra for the $\mathrm{WSe}_{2}$ and $\mathrm{MoS}_{2}$ monolayers are shown in Supplementary Figs. S1 and S2, respectively. Comparing our characterization spectra with the recent report of monolayer- $\mathrm{WSe}_{2}$ and monolayer- $\mathrm{WS}_{2}$ on sapphire ${ }^{3}$, they are similar. These are clear indications that the samples are of high monolayer quality.

\section{Temperature-Dependent Spectroscopic Ellipsometry Measurements and Absorption}

Coefficient. The J. A. Woollam Co., Inc. spectroscopic ellipsometer with photon energy of 1.2-3.4 eV has been used to measure the ellipsometry parameters $\Psi$ (the ratio between the amplitude of $p$-and $s$-polarized reflected light) and $\Delta$ (the phase difference between of $p$-and $s$-polarized reflected light) in a high vacuum chamber with a base pressure of $1 \times 10^{-}$ ${ }^{9}$ mbar. The sapphire substrate (bulk $\mathrm{Al}_{2} \mathrm{O}_{3}$ ) is also measured under the same conditions. The dielectric coefficient of the $\mathrm{WSe}_{2}\left(\mathrm{MoS}_{2}\right)$ monolayer was extracted by fitting the parameters $\Psi$ and $\Delta$ utilizing an air/ $/ \mathrm{WSe}_{2} / \mathrm{Al}_{2} \mathrm{O}_{3}$ or air/ $\mathrm{MoS}_{2} / \mathrm{Al}_{2} \mathrm{O}_{3}$ multilayer model, where the monolayer-WSe $2\left(\mathrm{MoS}_{2}\right)$ consists of a homogeneously uniform medium ${ }^{5-6}$ and a composite heterointerface component.

The ellipsometry parameters are defined as ${ }^{7}$ 


$$
\tan \Psi \exp (i \Delta) \equiv \frac{r_{p}}{r_{s}}
$$

where $r_{\mathrm{p}(\mathrm{s})}$ is the reflectivity of the $p-\left(s^{-}\right)$polarized light. Using the Fresnel equations, the quantities can be defined as

$$
r_{p}^{i j}=\frac{n_{j} \cos \theta_{i}-n_{i} \cos \theta_{j}}{n_{j} \cos \theta_{i}+n_{i} \cos \theta_{j}}
$$

and

$$
r_{s}^{i j}=\frac{n_{j} \cos \theta_{i}-n_{i} \cos \theta_{j}}{n_{j} \cos \theta_{i}+n_{i} \cos \theta_{j}}
$$

Here $n$ and $\theta$ represent the complex refractive index and the angle of incident, respectively. The $i$ and $j$ represent the two materials in each layer. The dielectric function $\varepsilon(\omega)=\varepsilon_{1}(\omega)+i \varepsilon_{2}(\omega)$ of material can be obtained using

$$
\sqrt{\varepsilon(\omega)}=n(\omega)
$$

where $\omega$ is the photon frequency.

The reflectivity of $\mathrm{WSe}_{2}$ film on substrate can be expressed as ${ }^{8}$,

$$
r_{m u l t i}=\frac{r_{a m b, W S_{e 2}}+r_{W S_{e 2}, s u b} \exp \left(i 2 \delta_{W S_{e 2}}\right)}{1+r_{a m b, W S_{e 2}} \cdot r_{W S_{e 2}, \text { sub }} \exp \left(i 2 \delta_{W S_{e 2}}\right)}
$$

where

$$
\delta_{W S_{e 2}}=\frac{2 \pi d_{W S_{e 2}}}{\lambda} \sqrt{n_{W S_{e 2}}^{2}-n_{a m b}^{2} \sin ^{2} \theta}
$$

Subscripts multi and amb represent the $\mathrm{WSe}_{2}$ on $\mathrm{Al}_{2} \mathrm{O}_{3}$ substrate multilayer system and the ambient, respectively, while $\delta_{\mathrm{WSe} 2}$ is the change in light phase as it reflects off the $\mathrm{WSe}_{2}$ film, and $d_{\mathrm{WSe} 2}$ is the thickness of the $\mathrm{WSe}_{2}$ film. $d_{\mathrm{WSe} 2}\left(d_{\mathrm{MoS} 2}\right)$ is used $0.7 \mathrm{~nm}$ for the average of thickness of $\mathrm{WSe}_{2}\left(\mathrm{MoS}_{2}\right)$ monolayer grown by CVD. For the refractive index of the substrate, the spectroscopic ellipsometry were measured separately. The 
temperature-dependent ellisometric measurements are performed at 70-degree incident angle for temperature-dependent measurements. The dielectric coefficient of $\mathrm{WSe}_{2}$ $\left(\mathrm{MoS}_{2}\right)$ monolayer is obtained through direct function inversion of Equation S4 after fitting.

First-Principles Calculations. First-principles calculations were performed using density-functional theory-based Vienna ab initio simulation package (VASP 5.4.4.18) 9-10. $^{\text {. }}$ The exchange-correlation functional and electron-ion interaction were treated by PerdewBurke-Ernzerhof (PBE) format functional and the projector augmented wave (PAW) potentials ${ }^{11-12}$, respectively. The cutoff energy of the electronic plane-wave basis has been set to $500 \mathrm{eV}$. A vacuum layer with a thickness of $20 \AA$ was inserted normal to the $\mathrm{MoS}_{2}$ and $\mathrm{WSe}_{2}$ monolayer surfaces. The Brillouin zone was sampled using $\Gamma$-centered $12 \times 12 \times 1$ k-point meshes. All 2D structures were optimized until the energy and force on each atom were converged smaller than $1.0 \times 1.0^{-8} \mathrm{eV}$ and $0.01 \mathrm{eV} / \AA$, respectively. Spinorbita coupling is also considered in the calculations. Based on these settings, the calculated lattice constants of the $\mathrm{MoS}_{2}$ and $\mathrm{WSe}_{2}$ monolayer are 3.184 and $3.325 \AA$, respectively, and the corresponding PBE band gaps are 1.67 and $1.56 \mathrm{eV}$, respectively. These are in good agreement with previous studies ${ }^{13-14}$. The optical spectra were calculated using Bethe-Salpeter equation (GW-BSE) (electron-hole interaction) on the top of the $\mathrm{G}_{0} \mathrm{~W}_{0}$ with the Tamm-Dancoff approximation ${ }^{15}$, of which, 216 empty bands were included. The energy cutoff for the response function was set to be $220 \mathrm{eV}$, and 12 highest valence bands and 8 lowest conduction bands were used as the basis for the excitonic eigenstates. 


\section{Band Structure of Monolayer-MoS ${ }_{2}$ With and Without the Effects of Spin-Orbit}

Coupling. To compare the band structures of monolayer-MoS $\mathrm{S}_{2}$ with and without the effects of spin-orbit coupling, the effects are as displayed in Supplementary Figs. S5a and 5b. Calculations show that by omitting the effects of spin-orbit coupling, the electronic structures are similar for both $\mathrm{MoS}_{2}$ and $\mathrm{WSe}_{2}$. This agrees with previous theoretical studies ${ }^{14,16-17}$. As displayed in Fig. S5b, without spin-orbit effects, monolayer- $\mathrm{MoS}_{2}$ has a direct bandgap of $\sim 1.68 \mathrm{eV}$ at the $\mathrm{K} / \mathrm{K}^{\prime}$-point of the Brillouin zone. At the region near the $\Gamma$ - and M-point, the bands are observed to be generally gentle.

With the introduction of SOC effects, we note that the band structure of monolayer-MoS $\mathrm{S}_{2}$ becomes significantly different. There are noticeable occurrences of spin-orbit splits in the band structures - especially in the highest occupied and lowest unoccupied bands. In particular, the appearance of exciton peaks $\mathrm{A}$ and $\mathrm{B}$ in monolayer- $\mathrm{MoS}_{2}$ (main text Fig. 1d) is the result of spin-orbit-splitting taking place at the VBM near the $\mathrm{K} / \mathrm{K}^{\prime}$-point (Fig. $\mathrm{S} 5 \mathrm{a})^{17}$. The spin-orbit coupling induced split of the valence band at the K-point of monolayer- $\mathrm{MoS}_{2}$ is $\sim 148 \mathrm{meV}$ - in good agreement with the position differences between peaks A and B of the $\mathrm{MoS}_{2}$ ellipsometric data (main text Fig. 1d). As for the conduction band spin-split at the $\mathrm{K} / \mathrm{K}^{\prime}$-points, it is significantly smaller at $\sim 3 \mathrm{meV}-$ in good agreement with previous computational studies ${ }^{16-17}$. The energy band above the CBM at the $\mathrm{K} / \mathrm{K}$ '-point (Fig. S5a) is located at $\sim 2.99 \mathrm{eV}$ above the $\mathrm{VBM}$ for $\mathrm{MoS}_{2}$.

Band Nesting Effects in 2D-TMDs. By analyzing the energy difference between the lowest unoccupied band and the highest occupied band in main text Fig. 4a and 
Supplementary Fig. S5a, $E_{\mathrm{c}}-E_{\mathrm{v}}(\approx 2.60 \mathrm{eV})$, alongside their gradients in this Brillouin zone region, notice that the gradient is generally low. Based on the criteria ${ }^{18}$ :

$$
\left|\nabla_{k}\left(E_{C}-E_{v}\right)\right| \ll 1 \mathrm{eV} /(2 \pi / a),
$$

this is the region where band-nesting occurs, and it corresponds to the strong optical response $\mathrm{C}$ known as a band nesting structure in the energy region of $\sim 2.5-2.6 \mathrm{eV}$ found in both monolayer-WSe 2 and $\mathrm{MoS}_{2}$.

Estimation of Exciton Binding Energy. Based on a previous experimental study, the binding energy of exciton $\mathrm{A}$ in monolayer-WSe $\mathrm{W}_{2}$ has been measured to be $\sim 0.37 \mathrm{eV}^{19}$. By combining this reference data with our experimentally determined position of exciton A (main text Fig. 1f), the bandgap of our monolayer- $\mathrm{WSe}_{2}$ sample can be estimated as $\sim 2.13 \mathrm{eV}$. In comparison with the computationally derived band structure of monolayer$\mathrm{WSe}_{2}$ with a calculated bandgap of $\sim 1.3 \mathrm{eV}$ (main text Fig. $4 \mathrm{a}$ ), the experimental result is significantly higher. Hence, an energy shift of $0.83 \mathrm{eV}$ is made to the calculated band structure data to account for the underestimation in the gaps of semiconductors and insulators by the PBE calculations ${ }^{12}$. With this energy shift applied to the calculated band structure and comparing it with the experimental data, the binding energy of exciton B is estimated to be $\sim 0.42 \mathrm{eV}$, in good agreement with that of exciton $\mathrm{A}^{19}$. Interestingly, the binding energy of resonant exciton $\mathrm{X}$ is estimated to be $\sim 0.8 \mathrm{eV}$. Previous theoretical studies of high-energy resonant excitons in graphene estimated the binding energy at $\sim 0.6 \mathrm{eV}^{20}$. The large binding energy and high-energy position of resonant exciton found in graphene serves as an appropriate reference in our analysis of resonant exciton X. 


\section{Supplementary References}

1. Stier, A. V.; Wilson, N. P.; Velizhanin, K. A.; Kono, J.; Xu, X.; Crooker, S. A., Magnetooptics of Exciton Rydberg States in a Monolayer Semiconductor. Phys. Rev. Lett. 2018, 120, 057405.

2. Kim, H.; Ahn, G. H.; Cho, J.; Amani, M.; Mastandrea, J. P.; Groschner, C. K.; Lien, D.-H.; Zhao, Y.; Ager, J. W.; Scott, M. C.; Chrzan, D. C.; Javey, A., Synthetic $\mathrm{WSe}_{2}$ Monolayers with High Photoluminescence Quantum Yield. Sci. Adv. 2019, 5, eaau4728.

3. Huang, J.-K.; Pu, J.; Hsu, C.-L.; Chiu, M.-H.; Juang, Z.-Y.; Chang, Y.-H.; Chang, W.-H.; Iwasa, Y.; Takenobu, T.; Li, L.-J., Large-Area Synthesis of Highly Crystalline $\mathrm{WSe}_{2}$ Monolayers and Device Applications. ACS Nano 2014, 8, 923-930.

4. $\quad$ Yin, X.; Wang, Q.; Cao, L.; Tang, C. S.; Luo, X.; Zheng, Y.; Wong, L. M.; Wang, S. J.; Quek, S. Y.; Zhang, W.; Rusydi, A.; Wee, A. T. S., Tunable Inverted Gap in Monolayer Quasi-Metallic $\mathrm{MoS}_{2}$ Induced by Strong Charge-Lattice Coupling. Nat. Commun. 2017, 8, 486.

5. $\quad$ Yin, X.; Majidi, M. A.; Chi, X.; Ren, P.; You, L.; Palina, N.; Yu, X.; Diao, C.; Schmidt, D.; Wang, B.; Yang, P.; Breese, M. B. H.; Wang, J.; Rusydi, A., Unraveling How Electronic and Spin Structures Control Macroscopic Properties of Manganite UltraThin Films. NPG Asia Mater. 2015, 7, e196.

6. Yin, X.; Zeng, S.; Das, T.; Baskaran, G.; Asmara, T. C.; Santoso, I.; Yu, X.; Diao, C.; Yang, P.; Breese, M. B. H.; Venkatesan, T.; Lin, H.; Ariando; Rusydi, A., Coexistence of Midgap Antiferromagnetic and Mott States in Undoped, Hole- and Electron-Doped Ambipolar Cuprates. Phys. Rev. Lett. 2016, 116, 197002.

7. Fujiwara, H., Spectroscopic Ellipsometry: Principles and Applications. John Wiley \& Sons Ltd, Tokyo, Japan, 2003.

8. Harbecke, B., Coherent and Incoherent Reflection and Transmission of Multilayer Structures. Appl. Phys. B. 1986, 39, 165-170.

9. Kresse, G.; Hafner, J., Ab Initio Molecular Dynamics for Open-Shell Transition Metals. Phys. Rev. B 1993, 48, 13115-13118.

10. Kresse, G.; Hafner, J., Ab Initio Molecular Dynamics for Liquid Metals. Phys. Rev. B 1993, 47, 558-561.

11. Blöchl, P. E., Projector Augmented-Wave Method. Phys. Rev. B 1994, 50, 1795317979.

12. Perdew, J. P.; Burke, K.; Ernzerhof, M., Generalized Gradient Approximation Made Simple. Phys. Rev. Lett. 1996, 77, 3865-3868.

13. Mak, K. F.; Lee, C.; Hone, J.; Shan, J.; Heinz, T. F., Atomically Thin $\mathrm{MoS}_{2}$ : A New Direct-Gap Semiconductor. Phys. Rev. Lett. 2010, 105, 136805.

14. Ramasubramaniam, A., Large Excitonic Effects in Monolayers of Molybdenum and Tungsten Dichalcogenides. Phys. Rev. B 2012, 86, 115409.

15. Paier, J.; Marsman, M.; Kresse, G., Dielectric Properties and Excitons for Extended Systems from Hybrid Functionals. Phy. Rev. B 2008, 78, 121201. 
16. Kośmider, K.; González, J. W.; Fernández-Rossier, J., Large Spin Splitting in the Conduction Band of Transition Metal Dichalcogenide Monolayers. Phys. Rev. B 2013, $88,245436$.

17. Zhu, Z. Y.; Cheng, Y. C.; Schwingenschlögl, U., Giant Spin-Orbit-Induced Spin Splitting in Two-Dimensional Transition-Metal Dichalcogenide Semiconductors. Phys. Rev. B 2011, 84, 153402.

18. Carvalho, A.; Ribeiro, R. M.; Castro Neto, A. H., Band Nesting and the Optical Response of Two-Dimensional Semiconducting Transition Metal Dichalcogenides. Phys. Rev. B 2013, 88, 115205.

19. He, K.; Kumar, N.; Zhao, L.; Wang, Z.; Mak, K. F.; Zhao, H.; Shan, J., Tightly Bound Excitons in Monolayer WSe 2 . Phys. Rev. Lett. 2014, 113, 026803.

20. Yang, L.; Deslippe, J.; Park, C.-H.; Cohen, M. L.; Louie, S. G., Excitonic Effects on the Optical Response of Graphene and Bilayer Graphene. Phys. Rev. Lett. 2009, 103, 186802. 